Kansas State University Libraries

New Prairie Press

Conference on Applied Statistics in Agriculture

2014 - 26th Annual Conference Proceedings

\title{
MODELING SLEEP AND WAKE BOUTS IN DROSOPHILA MELANOGASTER
}

Gayla R. Olbricht

Missouri University of Science and Technology, olbrichtg@mst.edu

V. A. Samaranayake

Missouri University of Science and Technology

Sahitya Injamuri

Missouri University of Science and Technology

Luyang Wang

Missouri University of Science and Technology

Courtney Fiebelman

Missouri University of Science and Technology

See next page for additional authors

Follow this and additional works at: https://newprairiepress.org/agstatconference

Part of the Agriculture Commons, and the Applied Statistics Commons

(c) $($ ) $\ominus$

This work is licensed under a Creative Commons Attribution-Noncommercial-No Derivative Works 4.0 License.

\section{Recommended Citation}

Olbricht, Gayla R.; Samaranayake, V. A.; Injamuri, Sahitya; Wang, Luyang; Fiebelman, Courtney; and Thimgan, Matthew S. (2014). "MODELING SLEEP AND WAKE BOUTS IN DROSOPHILA MELANOGASTER," Conference on Applied Statistics in Agriculture. https://doi.org/10.4148/2475-7772.1003

This Event is brought to you for free and open access by the Conferences at New Prairie Press. It has been accepted for inclusion in Conference on Applied Statistics in Agriculture by an authorized administrator of New Prairie Press. For more information, please contact cads@k-state.edu. 


\section{Author Information}

Gayla R. Olbricht, V. A. Samaranayake, Sahitya Injamuri, Luyang Wang, Courtney Fiebelman, and Matthew S. Thimgan 


\title{
MODELING SLEEP AND WAKE BOUTS IN DROSOPHILA MELANOGASTER
}

\author{
Gayla R. Olbricht ${ }^{1}$, V.A. Samaranayake ${ }^{1}$, Sahitya Injamuri ${ }^{2}$, Luyang Wang ${ }^{1}$, Courtney \\ Fiebelman $^{2}$, Matthew S. Thimgan ${ }^{2}$
}

${ }^{1}$ Department of Mathematics and Statistics, Missouri University of Science and Technology, 400 W. $12^{\text {th }}$ Street, Rolla, MO 65409; ${ }^{2}$ Department of Biological Sciences, Missouri University of Science and Technology, 400 W. $12^{\text {th }}$ Street, Rolla, MO 65409

\begin{abstract}
Adequate sleep restores vital processes required for health and well-being; but the function and regulation of sleep is not well understood. Unfortunately, a definition of adequate sleep is unclear. On an hours-long timescale, consolidated and cycling sleep results in better health and performance outcomes. At shorter timescales, older studies report conflicting results regarding the relationship between sleep and wake bout durations. One approach to this problem has been to simply analyze the distribution of bout durations. While informative, this method eliminates the time relationship between bouts, which may be important. Here, we develop a model that describes the relationship between sleep and wake bout durations using the model organism, Drosophila melanogaster, which exhibits behavioral and molecular homology to human sleep. We present an exploratory analysis of the data to gain a better understanding of the sleep bout duration distribution by considering a broader range of potential distributions than considered in previous studies. We use the results of the distribution analysis to develop a model for sleep bout durations in the fly based upon their past sleep and wake history and find that this relationship should not be ignored.
\end{abstract}

Keywords: Sleep, Drosophila melanogaster, sleep-wake transitions, Weibull distribution, Modeling

\section{Introduction}

Sleep is an essential part of life that is conserved across animal species. Lack of adequate sleep can result in many health consequences and increased mortality (Kripke et al., 2002). While the effects vary amongst species, sleep deprivation in humans has been shown to have numerous impacts including decreased cognitive performance (Van Dongen et al., 2004), increased Body Mass Index (BMI) (Taheri et al., 2004) and negative cardiovascular effects (Dettoni et al., 2012). Such negative effects are seen across species: prolonged sleep deprivation can lead to death in rats (Rechtschaffen et al., 1983), flies (Shaw et al., 2002), and even humans (Schenkein and 
Montagna, 2006). Although these findings highlight the importance of sleep, its function and how it is regulated are topics of ongoing research, as they are not fully understood.

In mammals, sleep has been modeled to be governed by two primary interacting processes. The first describes the approximately 24 hour cycling that occurs in most organisms, known as circadian rhythms or Process C. Process $\mathrm{C}$ gates the timing of sleep over a 24 hour period. The second is Process $\mathrm{S}$, the homeostatic process that involves the build-up of sleep pressure with increased wakefulness. These two systems interact to direct the timing and amount of sleep (Borbely, 1982). While many advances have been made to understand Process C, little is known about Process S. At the molecular level, sleep-wake cycles are also influenced by the integration of hundreds to thousands of interacting genetic and environmental inputs. To reduce some of this complexity, the fruit fly, Drosophila melanogaster, can be used as a model organism to understand the mechanisms that underlie sleep and translate these findings to sleep in mammals (Hendricks et al., 2000; Shaw et al., 2000; Sehgal and Mignot, 2011).

Drosophila research has played an important role in understanding circadian rhythms (Hardin, 2011) and Drosophila have been shown to display many molecular, behavioral, and genetic similarities to mammalian sleep (Shaw et al., 2000; Hendricks et al., 2000). There are many benefits to using Drosophila as a model organism. One prominent benefit is that molecular components of the circadian clock can be targeted and disrupted to eliminate Process $\mathrm{C}$, thereby focusing on the investigation of Process S (Konopka and Benzer, 1971; Sehgal et al., 1994; Allada et al., 1998; Rutila et al., 1998). Also, experimental conditions can be easily designed to study the influence of light-dark conditions or food availability, for example, on sleep behavior Thimgan et al., 2010). Moreover, Drosophila can also be monitored over long periods of time, yet have a short enough life span to allow the assessment of sleep patterns on longevity.

To begin to understand the relationship between sleep and wake cycles, it is important to record the timing of sleep and wake bouts over extended periods of time to understand how the patterns comprise adequate sleep. In mammals, several studies have employed mathematical and statistical methods to investigate the distribution of sleep and wake bout durations obtained from electroencephalography (EEG) and electromyography (EMG) readings (Lo et al., 2002; Lo et al., 2004; Blumberg et al., 2005). These studies fit both exponential and power law distributions to sleep and wake bout durations separately. Their findings indicate that sleep bout durations follow an exponential distribution across several species such as mice, cats, and humans with the scale parameter increasing with body size (Lo et al., 2004). Some deviations from the exponential distribution are evident in the tail. Wake bout durations were initially reported to follow a power-law distribution (Lo et al., 2002). However, a later study showed the distribution 
to be exponential in newborn rats, changing to power-law with maturity (Blumberg et al., 2005). Chu-Shore et al. (2010) pointed out that the power-law can easily be mistaken for other distributions using the methods employed in the initial studies. One limitation of the studies in mammals is the short recording duration and thus the limited number of transitions available for statistical analysis.

A second drawback of studying bout distributions is that they do not account for the potential temporal relationship of wake and sleep bouts. Investigating the short-term dependency of past sleep and wake behavior on the current sleep bout could provide additional insights into the nature of the sleep-wake cycle. Some studies have investigated the relationship between the previous wake and current sleep bout durations, but report conflicting results about the strength and direction of the relationship (Aschoff et al., 1969; Webb \& Friedman, 1969; Mistlberger et al. 1983; Wever, 1984). These studies do not consider dependencies beyond the previous bout, which may be important as both the amount of sleep and wake just prior to sleep could influence the length of the current bout.

In this study, we focus on modeling sleep bout durations in wild-type (Canton $S$ ) fruit flies. We examine sleep bout distributions as well as develop a model for the short-term temporal relationship between wake and sleep bout durations. Using Drosophila enables monitoring over a longer period (4 days) than in the mammalian studies ( $<1$ day) and it is instructive to understand the sleep architecture of a wild type fly for comparative purposes to other organisms and potentially to mutant flies. We extend upon the previous statistical methods by exploring a broader range of distributions commonly used for modeling life-spans to see if they provide a better fit than the exponential distribution. Results from the distributional analysis are employed to develop a statistical model that describes the relationship between the previous two bout (one wake and one sleep) durations with current sleep bout duration. This modeling can shed light on the short-term temporal dynamics of sleep. Furthermore, the results from this model could potentially be used in future studies to cluster flies by their parameter estimates and investigate consequences of deviations from normal estimates on health and performance of the organism.

\section{Data Collection}

Sleep is commonly monitored in flies through the Drosophila Activity Monitoring system, since it is technologically impossible to obtain EEG readings in freely behaving flies (Andretic and Shaw, 2005). In this system, individual flies are placed in separate glass vials with food at one end and a cotton plug at the other end. All flies are fed the same diet consisting of yeast, corn syrup, molasses, sucrose, and agar. An infrared beam passing through each vile is used to detect 
movement. Empirically it has been determined that if a fly is inactive (does not break beam) for at least a five minute period it meets the behavioral criteria for sleep (Shaw et al., 2000).

Otherwise the fly is deemed awake. There is censoring in the sense that true sleep bouts less than five minutes are not detected as sleep. Either male or Virgin female wild type (Canton $S$ ) flies aged 2-5 days old were placed in the individual vials and placed in constant dark ( $24 \mathrm{hrs})$ conditions. After two days of acclimation to the constant dark conditions, sleep and wake status was then recorded each minute over a four-day period (5760 minutes) on 29 female and 24 male flies. Sleep behavior of male and female flies is known to be different and the two sexes are analyzed separately. Sleep (and wake) bouts durations are found by calculating the length of time the fly stays in the sleep (or wake) status across consecutive minutes. Figure 1 shows how the data are first converted to sleep $(=0)$ and wake $(=1)$ status each minute based on beam crossings and then bout durations are calculated. Female flies have an average sleep bout duration of 20 minutes $(\mathrm{SD}=37.4)$ and male flies have an average sleep bout duration of 24 minutes $(\mathrm{SD}=39.0)$.

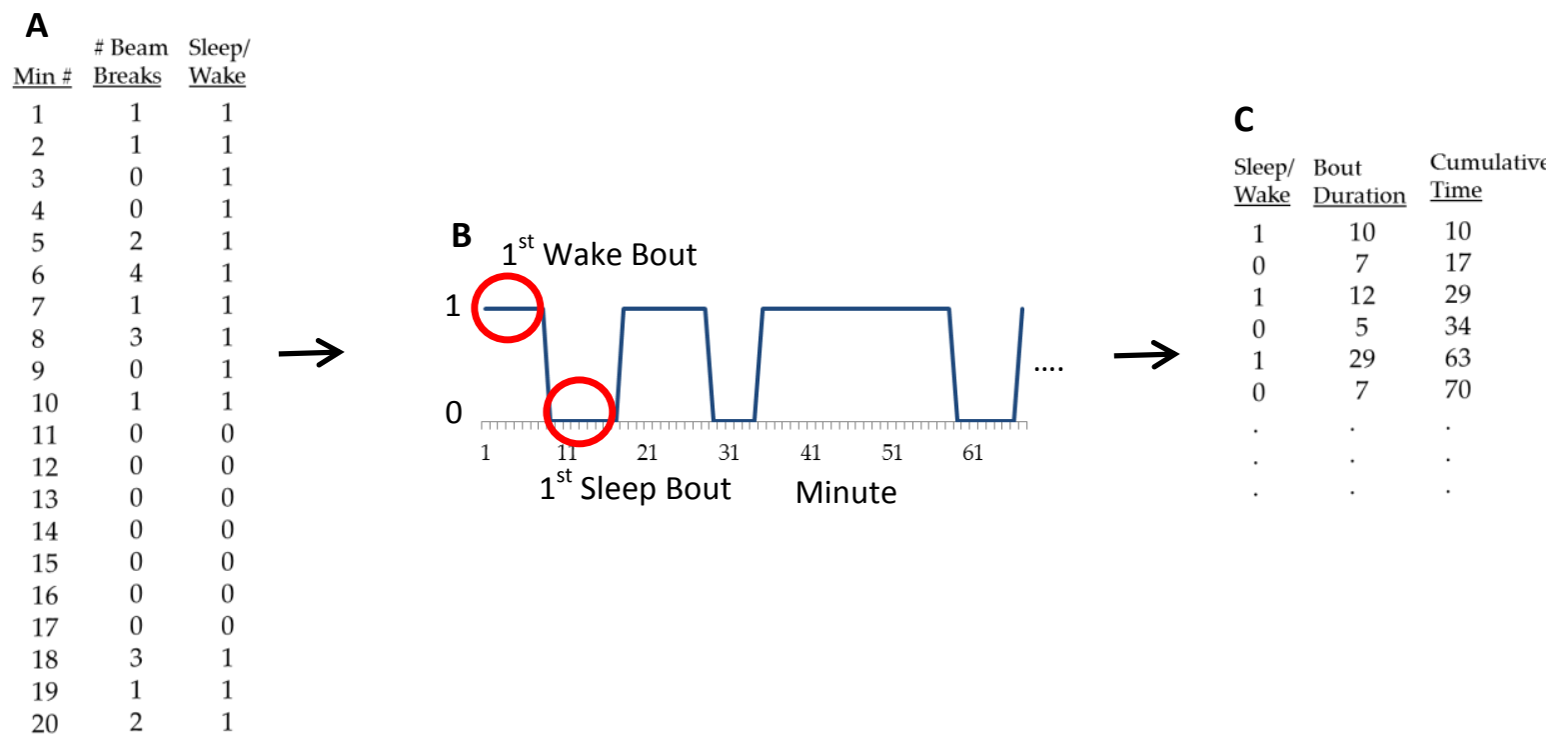

Figure 1. Illustration of data collection and conversion process. Panel A shows that the number of beam breaks are recorded each minute. For any period with 5 or more consecutive minutes of no beam crossings, the fly is given the sleep status (0) for those minutes. Otherwise, the fly is assigned the wake status (1). Panel B shows a graph of sleep/wake status over time, illustrating how sleep and wake bouts are defined by consecutive minutes of being in the same state. Panel C shows the results of bout duration calculations. 


\section{Statistical Analysis}

\subsection{Distribution of sleep bouts}

Methods. The first goal of our analysis is to investigate the distribution of sleep bout durations. Although previous studies have found that sleep bout durations follow an exponential distribution in mammals, there was deviation in the tail that indicates another distribution may be more appropriate. We examine a broader range of common life distributions that may provide a more suitable fit. We consider seven distributions: (1) exponential, (2) log-logistic, (3) lognormal, (4) Weibull, (5) threshold log-logistic, (6) threshold lognormal, and (7) threshold Weibull. The threshold distributions are considered due to the nature of the censored sleep bout durations that must by definition be at least 5 minutes long.

Let $Y_{i j}$ be the $i$ th bout duration for fly $j$ and $Y_{i j}^{0}$ be the subset of the bouts that correspond to the sleep status. Within each sex, we assume the sleep bout distribution is the same for all flies (i.e., $Y_{i j}^{0} \sim F(\boldsymbol{\theta})$ for all $j$ ), as was assumed and tested in the mammalian studies. For each of the seven distributions considered, maximum likelihood estimates were obtained for the parameters of each distribution using all $Y_{i j}^{0}$ within each sex. To compare fit of the distributions, the Akaike information criterion (AIC) was calculated as $A I C=2 p-2 \ln (L)$, where $p$ is the number of parameters of the distribution and $L$ is the maximized value of the likelihood function (Akaike, 1974). Smaller AIC values are indicative of a better fit and thus the distribution with the minimum AIC value was selected. The fit of the chosen distribution was further evaluated via a probability plot. All analysis was conducted using the Reliability Procedure in SAS v.9.3 software and JMP Pro 10 software.

Results. For both males and female Canton $S$ flies, the threshold Weibull distribution provided the best fit among the seven distributions that were tested using the AIC criterion (Figure 2, top panel). The exponential distribution yielded the worst fit. Probability plots of the cumulative distribution function (CDF) of the fitted threshold Weibull verses the sleep bout durations can be used to assess the fit of the threshold Weibull (Figure 2, middle panel). We see that the points approximately follow the theoretical solid line for both sexes, indicating the distribution is a good fit. The probability density function for the threshold Weibull is given below:

$$
f(x ; \alpha, \beta, \gamma)=\frac{\beta}{\alpha^{\beta}}(x-\gamma)^{\beta-1} \exp \left(-\left(\frac{x-\gamma}{\alpha}\right)^{\beta}\right), x>\gamma
$$

where $\alpha$ is the scale parameter, $\beta$ is the shape parameter, and $\gamma$ is the threshold parameter. The shape parameter allows flexibility in modeling the failure rate. When $\beta=1$, the distribution is 
exponential with a constant failure rate, but when $\beta<1$ or $\beta>1$ this is indicative of a decreasing or increasing failure rate, respectively. The threshold parameter indicates a shift of the Weibull distribution away from zero. The legends in the probability plots give the estimates for these three parameters for both sexes (Figure 2, middle panel). We see $\hat{\gamma}=4.999 \approx 5$, which is reasonable for these data since the sleep bout duration cannot be less than 5 and this supports the use of the threshold distribution to account for this feature in the data. The shape parameter estimate is less than one for both sexes $\left(\hat{\beta}^{\text {female }}=0.439, \hat{\beta}^{\text {male }}=0.585\right)$ indicating a decreasing failure rate. This means that the longer the fly sleeps the less likely the fly is to wake up. This may be explained by a high frequency of brief sleep periods (i.e., high "infant mortality"), but once the fly goes into an extended sleep period it stays asleep for a relatively long time. The scale parameter differs between the two sexes $\left(\hat{\alpha}^{\text {female }}=6.491, \hat{\alpha}^{\text {male }}=15.423\right)$. The improved fit of the threshold Weibull over the exponential suggests that it is important to allow for a nonconstant failure rate, as well as account for the five minute minimum sleep bout.

We also investigate variation in fit of the threshold Weibull distribution among individual flies within each sex. That is, we fit a threshold Weibull to each individual fly (i.e., $Y_{i j}^{0} \sim F\left(\boldsymbol{\theta}_{j}\right)$ for all i). Probability plots indicate that the threshold Weibull with a threshold parameter equal to five fits well for the individual flies, but there is more deviation in the male than female flies (Figure 2 , bottom panel). These results indicate that the threshold Weibull distribution provides a good fit for both individual and pooled data, but that parameters for individual flies may differ.

\subsection{Relationship between current and past bouts}

Methods. Using the results of the distributional analysis of sleep bouts, we propose a model relating the current sleep bout lengths to the previous two bout lengths (one sleep and one wake). The current sleep bout duration is assumed to follow a threshold Weibull distribution with the threshold parameter equal to 5. The log of the scale parameter is modeled as a linear function of the previous wake and sleep bout durations, while assuming a constant shape parameter. This model can be summarized as follows:

Let $Y_{i, j}^{0} \sim$ ThresholdWeibull $\left(\alpha_{j}, \beta_{j}, \gamma=5\right)$, where $\log \left(\alpha_{j}\right)=\beta_{0, j}+\beta_{1, j} Y_{i-1, j}^{1}+\beta_{2, j} Y_{i-2, j}^{0}$. 
Female

Distribution

Threshold Weibull

Threshold Loglogistic

Loglogistic

Lognormal

Weibull

Exponential

Threshold Lognormal
AIC

20015.578

24843.094

41281.661

41511.032

44274.775

44338.833

No Convergence

Male

Distribution

Threshold Weibull

Threshold Lognormal

Lognormal

Loglogistic

Weibull

Exponential

Threshold Loglogistic
AIC

24652.131

29750.686

30405.361

30528.196

31581.429

31583.606

No Convergence
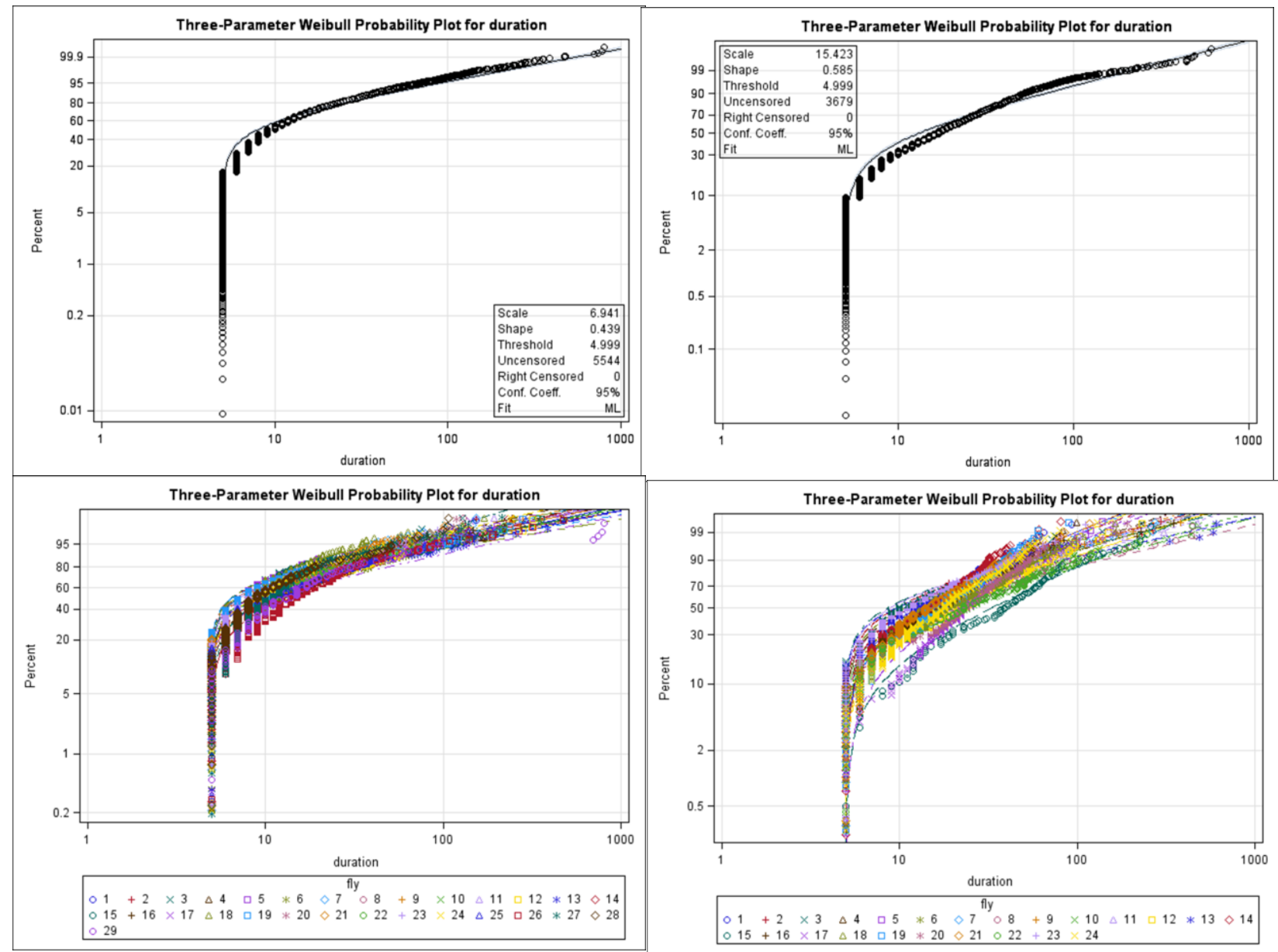

Figure 2. Results from distribution fitting. The left column gives results for female flies and the right column gives results for male flies. The top panel gives the AIC values for each of the seven different distributions, sorted in ascending order. The middle panel gives the probability plots from the threshold Weibull distribution by pooling all flies within each sex. The bottom panel gives the probability plots from the threshold Weibull distribution for individual flies. 
Recall that $Y_{i j}$ is the $i$ th bout duration for fly $j, Y_{i j}^{0}$ is the subset of the bouts that correspond to the sleep status, and $Y_{i j}^{1}$ is the subset of the bouts that correspond to the wake status. Note that the mean and median of the threshold Weibull are increasing functions of the scale parameter when the shape and threshold parameters are fixed. We assume that any dependencies in the current bout durations are accounted for by including the previous sleep and wake bouts in model. The regression parameters $\beta_{j}, \beta_{0 j}, \beta_{1 j}$, and $\beta_{2 j}$ are jointly estimated via maximum likelihood estimation. We fit a separate model for each fly and investigate the variation in the parameter estimates among flies of each sex. Three different hypotheses tests are conducted for each fly:

1. $H_{0 j}: \beta_{j}=1$ vs. $H_{A j}: \beta_{j} \neq 1$ (test for constant failure rate)

2. $H_{0 j}: \beta_{1 j}=0$ vs. $H_{A j}: \beta_{1 j} \neq 0$ (test for significance of past wake bout)

3. $H_{0 j}: \beta_{2 j}=0$ vs. $H_{A j}: \beta_{2 j} \neq 0$ (test for significance of past sleep bout).

Approximate $95 \%$ confidence intervals are employed to conduct these tests. Plots of the standardized residuals are used to check model fit.

\section{Results.}

The above three hypothesis tests were significant (null was rejected) for most of the 29 female flies. The shape parameter was significantly different from one for 24 of the female flies and all of these estimates were less than one, indicating a decreasing failure rate (Figure 4, top left). The slope parameter for past wake was significantly different from zero for 23 of the female flies and all of these estimates were negative, indicating that past wake has a negative relationship with current sleep (Figure 4, bottom left). The slope parameter for past sleep was significantly different from zero for 20 of the female flies and all of these estimates were positive, indicating that past sleep has a positive relationship with current sleep (Figure 4, bottom right). Interestingly, for the 24 male flies there were fewer significant and more varied results. The shape parameter was significantly different from one for 16 of the male flies. Six of these estimates were greater than one and 10 were less than one, indicating more variation in the type of failure rate among the male flies (Figure 4, top left). The slope parameter for past wake was significantly different from zero for six of the male flies, with five these estimates being negative as they were in females (Figure 4, bottom left). The slope parameter for past sleep was significantly different from zero for 10 of the male flies, with nine of these estimates being positive as they were for female flies (Figure 4, bottom right). Standardized residuals plots (not shown) indicated the threshold Weibull distribution provided a good fit. 

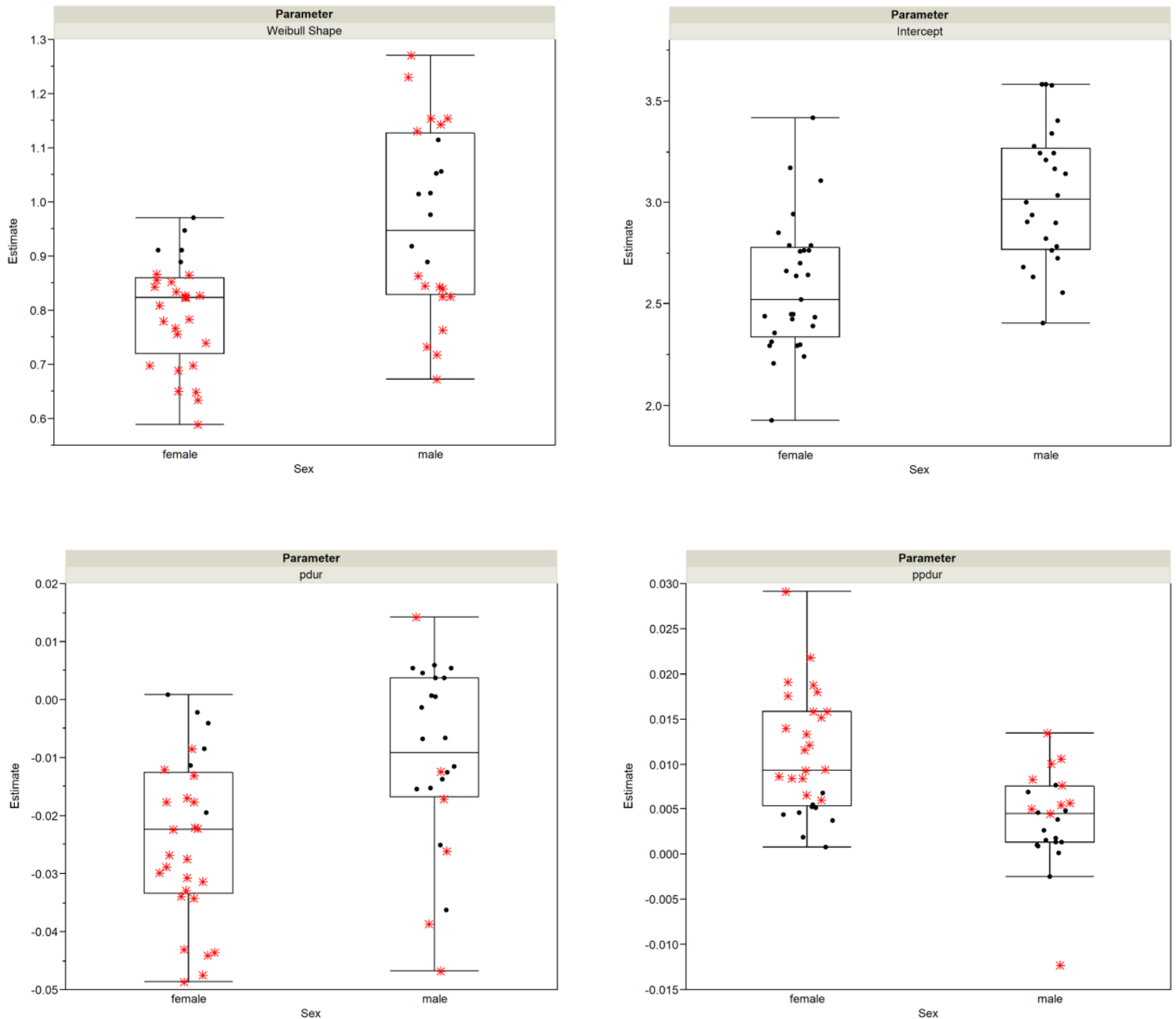

Figure 4. Boxplots of estimates for the shape parameter (top left), intercept (top right), and slope parameters for the past wake bout duration (bottom left) and the past sleep bout duration (bottom right). Points are the individual estimates. Red asterisks highlight flies with shape parameter values significantly different from one (top left) or slope parameters significantly different from zero (bottom panel). Separate boxplots are given for females and males. 
In summary, among the female flies we have evidence for a significant negative relationship between the past wake bout duration and current sleep bout duration, a positive relationship between the past sleep bout duration and current sleep bout duration, and a decreasing failure rate. Some male flies displayed these same characteristics, but some did not. Most notably, some male flies had an increasing failure rate, indicating a different type of sleep-wake behavior, and several did not show any significant relationship between the past wake and sleep bouts with the current sleep bout.

\section{Discussion and Summary}

In this work, we investigated the sleep-wake behavior of wild-type fruit flies. Specifically, we examined the distribution of sleep bout durations, as well as modeled the relationship between current sleep bouts and the previous sleep and wake bouts. Our work extends the approach from similar studies of bout distributions in mammals to the fruit fly. Our data has identified differences in the bout distribution between flies and previous work in mammals. These differences could indicate a difference in how bouts are distributed in the fly or indicate that the distributions considered in the mammalian studies may not be sufficient to adequately model the distribution of sleep bouts. In the wild type flies, the threshold Weibull model provided the best fit among seven different life-span distributions. Parameter estimates from this model, indicate that incorporating a threshold parameter to account for the 5 minute minimum sleep bout and allowing for a non-constant failure rate are important to consider. Future experiments involving fruit flies with mutations that disable the circadian rhythm will allow us to directly study Process S. Comparing distributions of the mutant flies to the wild-type flies can shed light on the differences in sleep with and without circadian rhythms.

Many previous studies did not address short-term dependencies in the sleep and wake bouts. Those that did found varied results, but many did indicate a negative relationship between past wake and current sleep bouts. The previous sleep bout was not considered in previous studies. Using the threshold Weibull model, we also found a negative relationship between past wake and current sleep bouts as well as a positive relationship between past sleep and current sleep bouts. These results were most consistent with the female flies, with male flies exhibiting more varied results. In the females, we found that the direction of the relationship (positive for past sleep and negative for past wake) held for up to 10 previous bouts. Although it may be ideal to include all flies in one model that accounts for correlation between bouts belonging to the same fly, it is computationally challenging to estimate the variance-covariance matrix of such a model. In addition, by obtaining individual parameter estimates for each fly we can investigate how these 
differences in these values relate to physical outcomes such as longevity and learning ability in future experiments.

Although our proposed model provides important information about short-term dependencies, long term dependencies should also be considered to better understand the sleep history. Specifically, although the nature of the relationships we observe indicate that the shorter the past wake bout and longer the past sleep bout the longer the current sleep bout, the direction of these relationships is unlikely to continue in the long term. These results along with other preliminary data we have analyzed are indicative of longer periods of wake and sleep that are briefly interrupted by the other state. We are investigating alternative models such as time series or regime switching models to study the long term behavior of the sleep-wake cycles.

\section{Acknowledgements}

We would like to thank the University of Missouri for funding and space. We would also like to thank the Center for Statistical and Computational Modeling of Biological Complexity at Missouri S\&T for funding contributing to these results.

\section{References}

Akaike H. (1974). A new look at the statistical model identification, IEEE Transactions on Automatic Control 19: 716-723.

Allada R, White NE, So WV, Hall JC, and Rosbash M. (1998). A mutant Drosophila homolog of mammalian Clock disrupts circadian rhythms and transcription of period and timeless, Cell 93: 791-804.

Andretic R and Shaw PJ. (2005). Essentials of sleep recordings in Drosophila: moving beyond sleep time, Methods Enzymol 393: 759-772.

Aschoff J, Gerecke U, Kureck A, Pohl H, Rieger P, et al. (1969). Interdependent parameters of circadian activity rhythms, Biochronometry: 3-29.

Blumberg MS, Seelke AMH, Lowen SB, and Karlsson KAE. (2005). Dynamics of sleep-wake cyclicity in developing rats, PNAS 102: 14860-14864.

Borbely, AA. (1982). A two process model of sleep regulation, Human Neurobiology 1: 195204. 
Chu-Shore J, Westover MB, and Bianchi MT. (2010). Power law verses exponential state transition dynamics: application to sleep-wake architecture, PLOS ONE 5: e14204.

Dettoni JL, Consolim-Colombo FM, Drager LF, Rubira MC, Cavasin de Souza SBP, et al. (2012). Cardiovascular effects of partial sleep deprivation in healthy volunteers, Journal of Applied Physiology 113: 232-236.

Hardin PE. (2011). Molecular genetic analysis of circadian timekeeping in Drosophila, Advances in Genetics 74: 141-173.

Hendricks JC, Finn SM, Panckeri KA, Chavkin J, Williams JA, et al. (2000). Rest in Drosophila is a sleep-like state, Neuron 25: 129-138.

Konopka RJ and Benzer S. (1971). Clock mutants of Drosophila melanogaster, Proc Natl Acad Sci USA 68: 2112-2116.

Kripke DF, Garfinkel L, Wingard DL, Klauber MR and Marler MR. (2002). Mortality associated with sleep duration and insomnia, Arch Gen Psychiatry 59: 131-136.

Lo C-C, Amaral LAN, Havlin S, Ivanov PC, Penzel T, et al. (2002). Dynamics of sleep-wake transitions during sleep, Europhysics Letters 57: 625-631.

Lo C-C, Chou T, Penzel T, Scammell TE, Strecker RE, et al. (2004). Common scale-invariant patterns of sleep-wake transitions across mammalian species, PNAS 101: 17545-17548.

Mistlberger RE, Bergmann BM, and Rechtschaffen A. (1983). Relationships among wake episode lengths, contiguous sleep episode lengths, and electroencephalographic delta waves in rats with suprachiasmatic nuclei lesions, Sleep 10: 12-24.

Rechtschaffen A, Gilliland MA, Bergmann BM, and Winter JB. (1983). Physiological correlates of prolonged sleep deprivation in rats, Science 221: 182-184.

Rutila JE, Suri V, Le M, So WV, Rosbash et al., (1998). CYCLE is a second bHLH-PAS clock protein essential for circadian rhythmicity and transcription of Drosophila period and timeless, Cell 93: 805-814.

SAS Institute Inc. (2011). SAS/QC ${ }^{\circledR} 9.3$ User's Guide. Cary, NC: SAS Institute Inc. 
SAS Institute Inc. (2012) JMP ${ }^{\circledR} 10$ Quality and Reliability Methods. Cary, NC: SAS Institute Inc.

Schenkein J and Montagna P. (2006). Self management of fatal familial insomnia. Part 1: what is FFI?, Med Gen Med 8: 65.

Sehgal A, Price JL, Man B, and Young MW. (1994). Loss of circadian behavioral rhythms and per RNA oscillations in the Drosophila mutant timeless, Science 263: 1603-1606.

Sehgal A and Mignot E. (2011). Genetics of sleep and sleep disorders, Cell 146: 194-207.

Shaw PJ, Cirelli C, Greenspan RJ, and Tononi G. (2000). Correlates of sleep and waking in Drosophila melanogaster, Science 287: 1834-1837.

Shaw PJ, Tononi G, Greenspan RJ, and Robinson DF. (2002). Stress response genes protect against lethal effects of sleep deprivation in Drosophila, Nature 417: 287-291.

Taheri S, Lin L, Austin D, Young T and Mignot E. (2004). Short sleep duration is associated with reduced leptin, elevated ghrelin, and increased body mass index, PLoS Med 1: e62.

Thimgan MS, Suzuki Y, Seugnet L, Gottschalk L, and Shaw PJ. (2010). The perilipin homologue, lipid storage droplet 2, regulates sleep homeostasis and prevents learning impairments following sleep loss, PLoS Biol 8.

Van Dongen HPA, Baynard MD, Maislin G, and Dinges DF. (2004). Systematic interindividual differences in neurobehavioral impairment from sleep loss: evidence of trait-like differential vulnerability, SLEEP 27: 423-433.

Webb WB and Friedman J. (1969). Length of sleep and length of waking interrelations in the rat, Psychonomic Science 17: 14-15.

Wever R. (1984). Properties of human sleep-wake cycles: parameters of internally synchronized free-running rhythms, Sleep 7: 27-51. 